\title{
New Beryllium results in halo stars from Keck/HIRES spectra
}

\author{
Ann Merchant Boesgaard ${ }^{1}$, Jeffrey A. Rich ${ }^{1}$, Emily M. Levesque ${ }^{1}$ \\ and Brendan P. Bowler ${ }^{1}$ \\ ${ }^{1}$ University of Hawaii, Institute for Astronomy \\ 2680 Woodlawn Drive, Honolulu, HI 96822, U.S.A. \\ email: boes@ifa.hawaii.edu, jrich@ifa.hawaii.edu, \\ emsque@ifa.hawaii.edu, bpbowler@ifa.hawaii.edu
}

\begin{abstract}
We have obtained high-resolution, high signal-to-noise Keck spectra to determine Be abundances in over 100 stars in the Galactic halo. The stellar metallicities range from $[\mathrm{Fe} / \mathrm{H}]=$ -0.50 to -3.50 . Using this large sample, we have examined the trends of Be with Fe and Be with $\mathrm{O}$. We find a real dispersion in $\mathrm{Be}$ at a given $[\mathrm{O} / \mathrm{H}]$ that indicates that $\mathrm{Be}$ may not be a good cosmochronometer. Our results indicate that the dominant production mechanism for Be changes as the Galaxy ages. In the early eras of the Galaxy, when massive stars become supernovae, Be is produced from the acceleration of energetic CNO atoms which bombard protons in the vicinity of supernovae. Later spallation reactions occur as high energy protons bombard CNO atoms in the interstellar gas. The change occurs near $[\mathrm{Fe} / \mathrm{H}]=-2.2$. We have found that Be is deficient in Li-deficient halo stars, which favors the blue straggler analog hypothesis.
\end{abstract}

Keywords. Stars: abundances, Population II, kinematics, late-type - Galaxy: halo

\section{Introduction}

The trio of the rare light elements provides excellent probes into many astrophysical issues: cosmology, the chemical evolution of the Galaxy, the origin of the light elements, element destruction in stars, stellar interiors and evolution, cosmic ray theory, hypernovae, etc. For many of these issues Be is the best probe. It has only one long-lived isotope, ${ }^{9} \mathrm{Be}$; the non-LTE effects are negligible; there is only one source for production (spallation); it is less fragile than the Li isotopes; and it has the potential to be a good cosmochronometer.

For our Be studies we have been using the Keck 10-m telescope (with more than 50\% more light-gathering power than 8-m telescopes) on the 4200-m Mauna Kea, which is above $40 \%$ of Earth's atmosphere. From 1993 to 2003 the CCD on HIRES (Vogt et al. 1994) had only $8 \%$ quantum efficiency near the Be II lines at 3130.4 and $3131.1 \AA$. However, after the upgrade of HIRES in 2004, the quantum efficiency on the new "blue chip" rose to $93 \%$. (The upgraded version now has 3 CCDs with $15-\mu$ m pixels.) We have been able to take UV spectra of stars almost 3 magnitudes fainter. However, signal-tonoise ratios of more than 100 are needed to find Be abundances in the most metal-poor stars, where the Be II lines are very weak.

Due to the benefits of large aperture and high altitude, we have been able to achieve some interesting results with the original version of HIRES. In our first comprehensive study of Be in halo stars, we found a linear relationship between $[\mathrm{Fe} / \mathrm{H}]$ and $\mathrm{A}(\mathrm{Be})(=\log$ $\mathrm{N}(\mathrm{Be}) / \mathrm{N}(\mathrm{H})+12.00$ ) with a slope of $+0.96 \pm 0.04$ (Boesgaard et al. 1999). We discovered a Be dip like the Li dip in the Hyades F dwarfs (Boesgaard \& King 2002), but unlike the 
Li decline in the Hyades G dwarfs, there is no decline in Be in the G dwarfs (Boesgaard \& King 2002). We followed that up with Be studies in F and $\mathrm{G}$ dwarfs in several other open clusters: Pleiades and $\alpha$ Per (Boesgaard et al. 2003a), Coma and UMa moving group (Boesgaard et al. 2003b), and Praesepe (Boesgaard et al. 2004a). In a large study of Li and $\mathrm{Be}$ in both field and cluster stars we found that the depletions of $\mathrm{Li}$ and $\mathrm{Be}$ are correlated in the stars in the Li-Be dip with a slope of $+0.38 \pm 0.03$ (Boesgaard et al. 2004b). This could result from rotationally induced mixing. By studying B in stars with large Be deficiencies, we were able to find a correlation between Be and $\mathrm{B}$ depletions (Boesgaard et al. 2005); the slope for that relationship is $0.22 \pm 0.05$.

\section{Observations with the upgraded HIRES}

The upgraded HIRES has a mosaic of 3 CCDs, each $2048 \times 2048$ pixels with a pixel size of $15 \mu \mathrm{m}$. Since September 2004, we have received 16 nights of Keck time with HIRES over a 41-month period to pursue research on Be. Only two of those nights were lost: one due to the closure of the road to the summit from a raging blizzard and one due to two deep undersea earthquakes measuring 6.7 and 6.0 on the Richter scale at just after $7 \mathrm{a}$.m. on the morning of our one-night run. They caused damage to both Keck telescopes and to the remote-observation rooms. On the other 14 nights, we obtained high signal-to-noise $(\mathrm{S} / \mathrm{N})$ spectra of over 100 stars for several different research goals. As Fig. 1 in Rich \& Boesgaard (2009) shows, it is important to achieve high $\mathrm{S} / \mathrm{N}$ in the most metal-poor stars due to the weakness of the Be II lines.

We determined the stellar parameters - $T_{\text {eff }}, \log g,[\mathrm{Fe} / \mathrm{H}]$, and microturbulence $(\xi)$ spectroscopically (see Rich \& Boesgaard 2009). The values ranged from 5550 to $6400 \mathrm{~K}$, 3.2 to 4.9 in $\log g,-0.5$ to -3.5 in $[\mathrm{Fe} / \mathrm{H}]$, and 0.9 to $1.5 \mathrm{~km} \mathrm{~s}^{-1}$ in $\xi$. The spectrum synthesis method was used to find abundances; an example is shown in Fig. 1, where both $\mathrm{Be}$ and $\mathrm{O}$ were varied to achieve the best fit. Oxygen abundances were found from three $\mathrm{OH}$ features. The two other $\mathrm{OH}$ features $(3139,3140 \AA)$ can be seen in Fig. 3 of Rich \& Boesgaard (2009). Each feature contains several OH transitions.

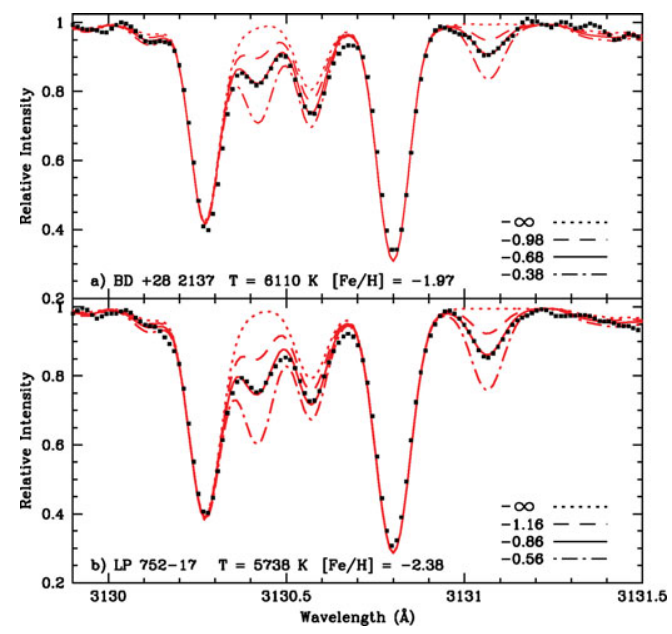

Figure 1. The spectrum synthesis fit for two of our stars: BD +282137 at $[\mathrm{Fe} / \mathrm{H}]=-1.97$ and LP $752-17$ at $[\mathrm{Fe} / \mathrm{H}]=-2.38$. The dots are the data points and the solid line is the best fit. The dot-dash line has twice as much Be and 0.1 dex more $\mathrm{O}$ than the best fit. The dashed line has half the $\mathrm{Be}$ and 0.1 dex less $\mathrm{O}$. The dotted line contain no Be and is another 0.1 dex lower in $\mathrm{O}$. 


\section{Abundances and trends}

Be trends with $\mathrm{Fe}$ and with $\mathrm{O}$. We have been able to fit the trends of Be with $\mathrm{Fe}$ and Be with $\mathrm{O}$ with straight lines in the log-log plot. We prefer a two slope fit for both. These fits are shown in Fig. 2. Data from Smiljanic et al. (2009) are included in the figure with $[\mathrm{Fe} / \mathrm{H}]$, but not in the figure with $[\mathrm{O} / \mathrm{H}]$ as they did not determine $\mathrm{O}$ abundances.

A change in slope would be expected. In the oldest stars, Be would be formed mostly in the vicinity of SN II by the acceleration of CNO nuclei into protons, etc. Thus Be would be proportional to the instantaneous number of supernovae and therefore proportional to $\mathrm{O}$ with a slope of $\leqslant 1$. In the younger stars, the dominant formation mechanism would be traditional GCR spallation from high-energy cosmic rays bombarding CNO in the interstellar gas. The number of $\mathrm{O}$ atoms would depend on the cumulative number of supernovae, while the number of energetic cosmic rays is proportional to the instantaneous rate of SN II. The abundance of spallation products is $\int N d N=k N^{2}$ giving a slope of $\leqslant 2$. Due to effects such as mass outflow during star formation, the predicted slopes of 1 and 2 would be modified to lower values.
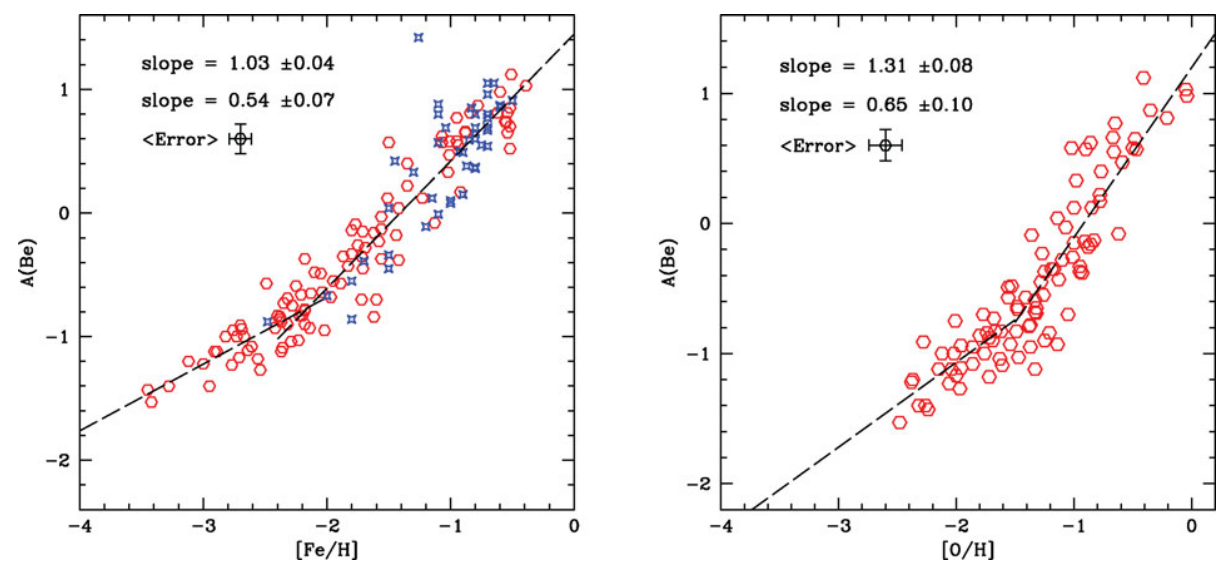

Figure 2. The two-slope fit of $\mathrm{A}(\mathrm{Be})$ with $[\mathrm{Fe} / \mathrm{H}]$ and $\mathrm{A}(\mathrm{Be})$ with $[\mathrm{O} / \mathrm{H}]$. The open hexagons are from this work and the skeletal squares are from Smiljanic et al. (2009).

Beryllium plateau? We find no evidence for a Be plateau at low metallicities comparable to the Li plateau. There may be a steady increase in $[\mathrm{Be} / \mathrm{Fe}]$ at the lowest values of $[\mathrm{Fe} / \mathrm{H}]$, but this may only reflect the paucity of stars (only 5) observed for Be with $[\mathrm{Fe} / \mathrm{H}]<$ -3.0 .

Beryllium spread. We do find evidence for a real spread in $\mathrm{A}(\mathrm{Be})$ at a given $[\mathrm{O} / \mathrm{H}]$ at the $4 \sigma$ level and probably at a given $[\mathrm{Fe} / \mathrm{H}]$ at the $3 \sigma$ level. We have done statistical tests using a prediction interval. We start with the null hypothesis that there is no spread in Be abundances in a certain Fe or $\mathrm{O}$ interval. We derive slope and offset values from the points outside the metallicity interval under consideration. We then use those data to predict the range of Be values inside our metallicity interval for a given confidence level, and we compare that with the actual data. For example, if there are 25 points in our metallicity interval, and 7 lie outside the $95 \%$ confidence level, we can then use the binomial theorem to determine the probability of this occurring by chance. We can try different confidence levels $(99 \%, 95 \%, 90 \%)$ and different size bins for $[\mathrm{Fe} / \mathrm{H}]$ and $[\mathrm{O} / \mathrm{H}]$ $( \pm 0.25, \pm 0.20, \pm 0.10)$. Fig. 3 shows an example of this. The probability that 7 of 25 stars fall outside the $95 \%$ confidence level by chance is $0.015 \%$. 


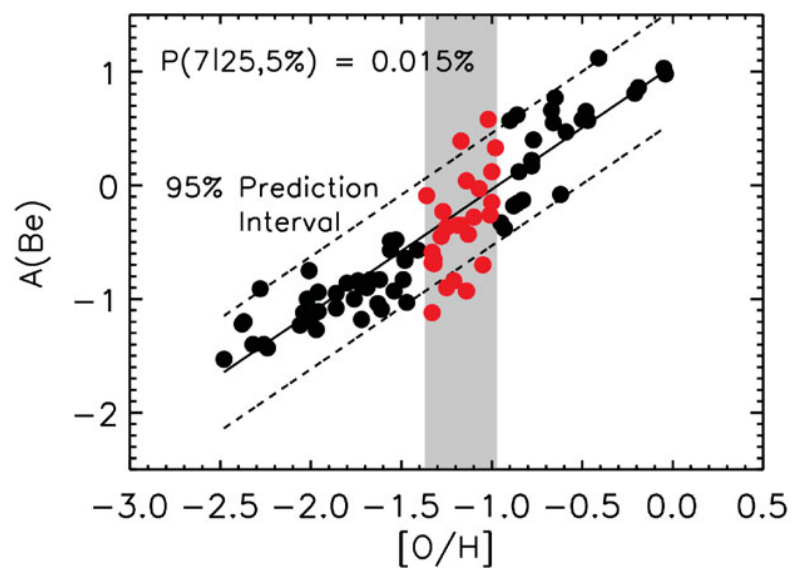

Figure 3. An example of the prediction-interval testing that we did to check for a spread in $\mathrm{Be}$ at a given value of $[\mathrm{O} / \mathrm{H}]$ or $[\mathrm{Fe} / \mathrm{H}]$.

There seems to be little evidence in our data sample of a different distribution of Be with $[\mathrm{O} / \mathrm{Fe}]$ for "accretive" vs. "dissipative" stars as determined by the criteria of Gratton et al. (2003). Although the errors were large for $[\mathrm{O} / \mathrm{Fe}]$ in the stars studied by Pasquini et al. (2005) and in $[\alpha / \mathrm{Fe}]$ in the stars for Smiljanic et al. (2009), both groups found intriguing differences in the two sets of stars. They concluded that Be can be used as a chronometer in a subset of stars. They find a large scatter in the dissipative stars and two sequences for the accretive. Our results can be seen in Fig. 4; both accretive and dissipative stars show a spread in $[\mathrm{O} / \mathrm{H}]$ and no separation of two sequences for the accretive stars. We found an intrinsic spread in $\mathrm{Be}$ at a given $\mathrm{Fe}$ or $\mathrm{O}$. In addition there is a spread in $[\mathrm{O} / \mathrm{Fe}]$ of more than $2 \sigma$ at a given $\mathrm{A}(\mathrm{Be})$. We conclude than Be may not fulfill its potential as a chronometer.
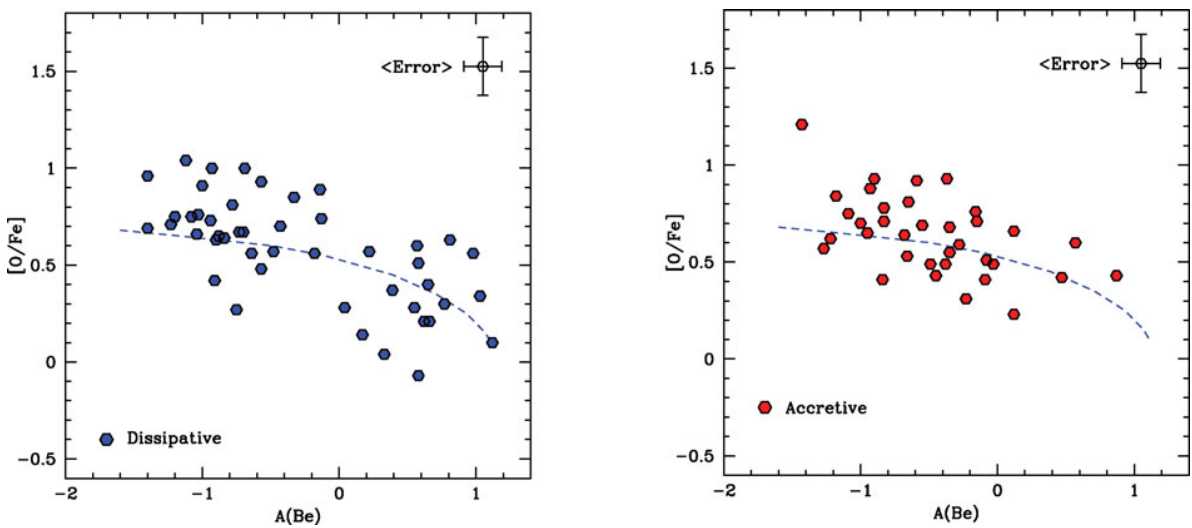

Figure 4. A plot of $[\mathrm{O} / \mathrm{Fe}]$ vs. A(Be) in stars in the "accretive" and dissipative" populations (as defined by Gratton et al. 2003). We see no evidence for a difference in the distribution between the two populations.

\section{Beryllium in ultra-Lithium-deficient stars}

Although most metal-poor stars have $\mathrm{A}(\mathrm{Li})$ values between $\sim 1.9$ to 2.5 , there are a few that are deficient in $\mathrm{Li}$ and fall below this plateau. This can be seen in Fig. 5 (left). 
These were dubbed "ultra-Li-deficient" stars by Ryan et al. (2001). There are at least two plausible explanations for the Li deficiencies. Ryan et al. (2001, 2002) suggest that they are blue-straggler analogs in which mass transfer or binary coalescence has occurred that destroyed the Li. Pinsonneault et al. $(1999,2002)$ hypothesize that they are a subset of (once) rapidly rotating stars which have depleted their $\mathrm{Li}$ as they spun down their rotation rate. These two ideas make different predictions about Be. In the blue straggler model, most or all of the Be would also be destroyed due to complete internal mixing. In the rotation model some or all of the Be would be preserved because Be is less fragile than Li.

Boesgaard \& Novicki (2006) and Boesgaard (2007) determined Be abundances in seven of the nine Li-deficient stars that are indicated in the left panel of Fig. 5. They found that the Li-deficient stars are also Be-deficient as can be seen the in right panel of Fig. 5 . Those Be deficiencies are larger than the predictions from the models of rotationallyinduced mixing by Pinsonneault et al. (1992). This result thus favors the blue-straggler model which predicts large Be deficiencies. Newer models by Pinsonneault et al. (2002) predict smaller Li depletions, and probably smaller Be depletions as well.
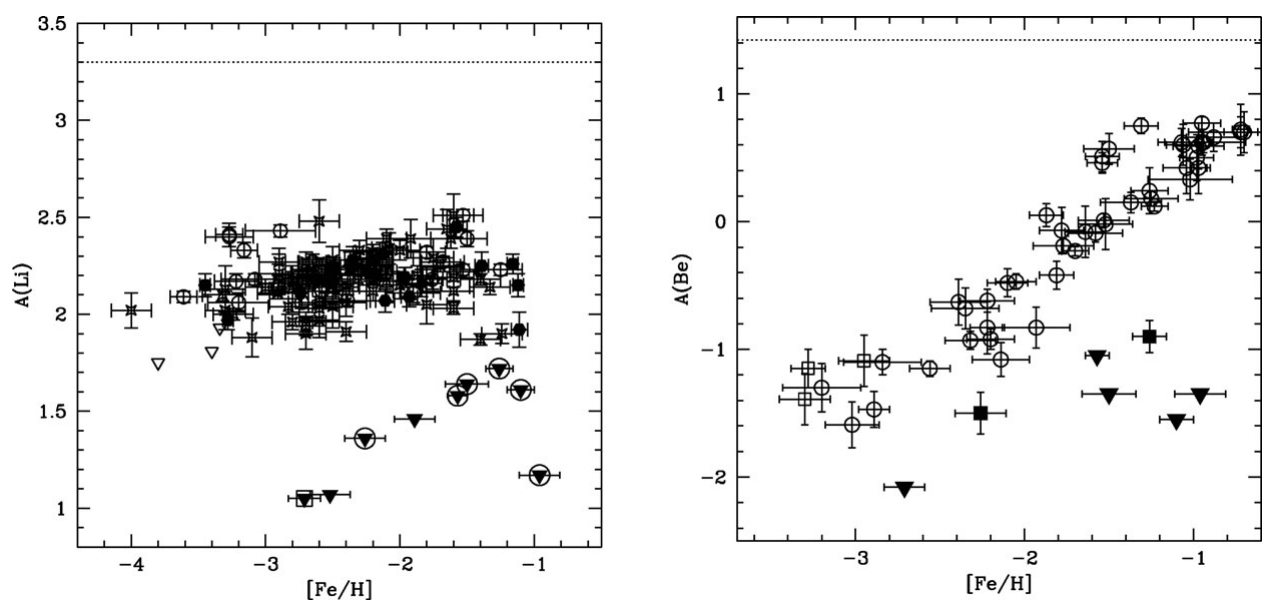

Figure 5. Left: The abundance of $\mathrm{Li}, \mathrm{A}(\mathrm{Li})=\log \mathrm{N}(\mathrm{Li})-\log \mathrm{N}(\mathrm{H})+12.00$, plotted against $[\mathrm{Fe} / \mathrm{H}]$ for halo dwarfs. The upper limits for the Li-deficient stars are indicated by downward triangles; those observed for Be have circles around them (Boesgaard 2007) or a square (Boesgaard \& Novicki 2006). See the caption on the similar figure in Figure 1 of Boesgaard (2007) for the detailed references. The horizontal dotted line is the meteoritic $\mathrm{Li}$ abundance, $\mathrm{A}(\mathrm{Li})=$ 3.30 (Grevesse \& Sauval 1998). Right: The Be abundances/limits of the ultra-Li-deficient stars shown in the context of Be abundances in Li-normal stars as a function of $[\mathrm{Fe} / \mathrm{H}]$. The filled squares are the two ultra-Li-deficient stars with detected Be, while the filled triangles represent the upper limits on four ultra-Li-deficient stars. Open circles are from Boesgaard et al. (1999), and other references are given in Boesgaard (2007). Open squares (low-metallicity stars) are from Primas et al. (2000a, 2000b). Individual error bars on both $\mathrm{A}(\mathrm{Be})$ and $[\mathrm{Fe} / \mathrm{H}]$ are shown. The horizontal dotted line is the meteoritic Be abundance, $\mathrm{A}(\mathrm{Be})=1.42$ (Grevesse \& Sauval 1998).

\section{Summary of Keck Be results}

Our findings from our Keck Be data include the following:

- There are correlated depletions of Li with Be and of Be with B. These observations are well-matched by the predictions of rotationally induced mixing. 
- There is a Be dip like the Li dip in the Hyades F stars and in other open clusters. However, for the $\mathrm{G}$ stars there is no decline in Be to mirror the decline in Li.

- We have found abundance trends between $\mathrm{A}(\mathrm{Be})$ and $[\mathrm{Fe} / \mathrm{H}]$ and between $\mathrm{A}(\mathrm{Be})$ and $[\mathrm{O} / \mathrm{H}]$ which can be well fit by one straight line or by two. The two-slope fits are preferred and expected if the dominant source of Be production changes during the course of Galactic evolution from core-collapse supernovae (bullets $=\mathrm{CNO}$ and targets $=\mathrm{p}, \mathrm{n}$ ) to GCR spallation (bullets $=\mathrm{p}, \mathrm{n}$ and targets $=\mathrm{CNO}$ ). There are also tight relationships between $[\mathrm{Fe} / \mathrm{H}]$ and $[\mathrm{O} / \mathrm{H}]$ and between $[\mathrm{O} / \mathrm{Fe}]$ and $[\mathrm{Fe} / \mathrm{H}]$.

- There appears to be a real spread in Be at a given $[\mathrm{Fe} / \mathrm{H}]$ and a given $[\mathrm{O} / \mathrm{H}]$. We find no difference in the relationships between $\mathrm{Be}$ and $[\mathrm{O} / \mathrm{Fe}]$ for the accretive sample and the dissipative sample. This would seem to indicate that Be cannot be relied on as a cosmochronometer.

- We have found Be abundances and upper limits in those halo stars where the upper limits on the $\mathrm{Li}$ abundances put them well-below the Li plateau. A plausible explanation is that these stars are analogs to blue stragglers and have destroyed most or all of their $\mathrm{Li}$ and Be though mass transfer or binary coalescence.

\section{Acknowledgements}

We gratefully acknowledge support from the National Science Foundation through grant AST-05-05899 to A. M. B.

\section{References}

Boesgaard, A. M. 2007, ApJ, 667, 1196

Boesgaard, A. M. \& King, J. R. 2002, ApJ, 565, 587

Boesgaard, A. M. \& Novicki, M. C. 2006, ApJ, 641, 1122

Boesgaard, A. M., Armengaud, E., \& King, J. R. 2003a, ApJ, 582, 410

Boesgaard, A. M., Armengaud, E., \& King, J. R. 2003b, ApJ, 583, 955

Boesgaard, A. M., Armengaud, E., \& King, J. R. 2004a, ApJ, 605, 864

Boesgaard, A. M., Armengaud, E., King, J. R., Deliyannis, C. P., \& Stephens, A. 2004b, ApJ, 613,1202

Boesgaard, A. M., Deliyannis, C. P., King, J. R., Ryan, S. G., Vogt, S. S., \& Beers, T. C. 1999, $A J, 117,1549$

Boesgaard, A. M., Stephens, A., \& Deliyannis, C. P. 2005, ApJ, 633, 398

Gratton, R. G., Carretta, E., Claudi, R, Lucatello, S., \& Barbien, M. 2003, A\&A, 404, 187

Grevesse, N. \& Sauval, A. J. 1998, Space Sci. Rev., 85, 161

Pasquini, L., Galli, D., Gratton, R. G., Bonifacio, P., Randich, S., \& Valle, G. 2005, A\&A (Letters), 436, 57

Pinsonneault, M. H., Deliyannis, C. P., \& Demarque, P. 1992, ApJS, 78, 179

Pinsonneault, M. H., Steigman, G., Walker, T. P., \& Narayanan, V. K. 2002, ApJ, 574, 398

Pinsonneault, M. H., Walker, T. P., Steigman, G., \& Narayanan, V. K. 1999, ApJ, 527, 180

Primas, F., Asplund, M., Nissen, P. E., \& Hill, V. 2000a, A $\mathscr{E} A$ (Letters), 364, 42

Primas, F., Asplund, M, Bonifacio, P., \& Hill, V. 2000b, A\&A, 362, 666

Rich, J. A. \& Boesgaard, A. M. 2009, ApJ, 701, 1519

Ryan, S. G., Beers, T. C., Kajino, T., \& Rosolankova, K. 2001, ApJ, 547, 231

Ryan, S. G., Gregory, S. G., Kolb, U., Beers, T. C., \& Kajino, T. 2002, ApJ, 571, 501

Smiljanic, R., Pasquini, L., Bonifacio, P., Galli, D., Gratton, R. G., Randich, S., \& Wolff, B. 2009, A\&BA, 499, 103

Vogt, S. S. et al. 1994, SPIE, 2198, 362 